\title{
The neural correlates of risk propensity in males and females using resting-state fMRI
}

\author{
Yuan Zhou ${ }^{1}$, Shu $L_{i}{ }^{1}$, John Dunn ${ }^{2}$, Huandong $L_{i}{ }^{3}$, Wen Qin ${ }^{4}$, Maohu Zhu ${ }^{3}$, Li-Lin Rao ${ }^{1}$, Ming Song ${ }^{3}$, \\ Chunshui $\mathrm{Yu}^{4 *}$ and Tianzi Jiang ${ }^{3,5,6 *}$ \\ ${ }^{1}$ Key Laboratory of Behavioral Science, Institute of Psychology, Chinese Academy of Sciences, Beijing, China \\ 2 The School of Psychology, The University of Adelaide, Adelaide, SA, Australia \\ ${ }^{3}$ Brainnetome Center and National Laboratory of Pattern Recognition, Institute of Automation, Chinese Academy of Sciences, Beijing, China \\ ${ }^{4}$ Department of Radiology, Tianjin Medical University General Hospital, Tianjin, China \\ ${ }^{5}$ Key Laboratory for Neurolnformation of Ministry of Education, School of Life Science and Technology, University of Electronic Science and Technology of China, \\ Chengdu, China \\ ${ }^{6}$ The Queensland Brain Institute, The University of Queensland, Brisbane, QLD, Australia
}

\section{Edited by:}

Carmen Sandi, École Polytechnique

Fédérale de Lausanne, Switzerland

\section{Reviewed by:}

Bettina Studer, University College

London, UK

Tatjana Aue, University of Geneva,

Switzerland

\section{*Correspondence:}

Chunshui Yu, Department of

Radiology, Tianjin Medical University

General Hospital, No. 154, Anshan

Road, Heping District, Tianjin

300052, China

e-mail: chunshuiyu@yahoo.cn;

Tianzi Jiang, National Laboratory of

Pattern Recognition, Brainnetome

Center, Institute of Automation,

Chinese Academy of Sciences,

No. 95, Zhongguancun East Road,

Haidian District, Beijing 100190,

China

e-mail: jiangtz@nlpr.ia.ac.cn
Men are more risk prone than women, but the underlying basis remains unclear. To investigate this question, we developed a trait-like measure of risk propensity which we correlated with resting-state functional connectivity to identify sex differences. Specifically, we used short- and long-range functional connectivity densities to identify associated brain regions and examined their functional connectivities in resting-state functional magnetic resonance imaging (fMRI) data collected from a large sample of healthy young volunteers. We found that men had a higher level of general risk propensity (GRP) than women. At the neural level, although they shared a common neural correlate of GRP in a network centered at the right inferior frontal gyrus, men and women differed in a network centered at the right secondary somatosensory cortex, which included the bilateral dorsal anterior/middle insular cortices and the dorsal anterior cingulate cortex. In addition, men and women differed in a local network centered at the left inferior orbitofrontal cortex. Most of the regions identified by this resting-state fMRI study have been previously implicated in risk processing when people make risky decisions. This study provides a new perspective on the brain-behavioral relationships in risky decision making and contributes to our understanding of sex differences in risk propensity.

Keywords: functional connectivity, functional magnetic resonance imaging, resting state, risk propensity, sex difference

\section{INTRODUCTION}

Most decisions involve risk, and the general willingness to take such risks is called risk propensity (Harrison et al., 2005). Men tend to have higher levels of risk propensity than women (Byrnes et al., 1999; Croson and Gneezy, 2009; Wang et al., 2009; Dohmen et al., 2011), a difference that is consistent across time and in a variety of contexts (Powell and Ansic, 1997). Despite these welldocumented sex differences, little is known regarding their neural correlates. Two previous studies have examined the activation of brain regions during a gambling task and risk-taking task but found inconsistent patterns of sex differences (Bolla et al., 2004; Lee et al., 2009). In one of these studies, task-reduced activity in the insula and right lateral orbitofrontal cortex (OFC) was associated with risk preference in women, but not in men (Lee et al., 2009). However, these studies as well as others which did not examine sex-related differences (e.g., Paulus et al., 2003; Hsu et al., 2005; Tobler et al., 2007; Christopoulos et al., 2009), investigated changes in brain activity associated with risky decision making tasks and so did not directly address the neural correlates of the enduring trait of risk propensity. How the neural correlates of this general disposition are differentially expressed in men and women is currently unknown. To address this question, it is necessary to find a stable measure of risk propensity and to correlate it with a similarly stable and enduring characteristic of neural activity.

Resting-state functional magnetic resonance imaging (fMRI) is an important approach in the study of human brain functions (Raichle, 2010). Resting-state functional connectivity (RSFC), which measures inter-regional correlations among spontaneous low-frequency $(<0.1 \mathrm{~Hz})$ fluctuations in the fMRI signal (Biswal et al., 1995), can be used to identify enduring and intrinsic properties of the brain (Fox and Raichle, 2007). As well as reflecting underlying anatomical connectivity (Park and Friston, 2013), RSFC has also been shown to correspond to the brain's functional architecture in response to external stimuli (Raichle and Mintun, 2006; Smith et al., 2009), as evidenced by findings that intrinsic resting-state brain activity can predict task-evoked brain activation during different cognitive tasks (Fox et al., 2006, 2007; Mennes et al., 2010; Liu et al., 2011; Mennes et al., 2011; Zou et al., 2013). RSFC has also been used to characterize functional brain networks correlated with individual differences in behavioral traits, such as personality, autistic trait and aggression (Di Martino et al., 2009; Hoptman et al., 2010; Adelstein et al., 2011). To our knowledge, only two studies have explored the neural correlates of risk propensity using RSFC (Cox et al., 2010; Han et al., 2012). Using a seed-based RSFC approach, these groups have 
associated the RSFCs of brain regions implicated in the evaluation of risk and reward (nucleus accumbens) as well as cognitive control and risk aversion (anterior insula and anterior cingulate cortex) with various risk-aversion measures. Both studies have suggested that individual differences in risk propensity are correlated with the brain's intrinsic functional architecture, which, in turn, may reflect experience-based recruitment of specific brain regions in the active processing of risk. However, these studies did not address the neural correlates of sex differences in risk propensity, and the seed-based approach employed limited their findings to specific regions.

To detect functional networks underlying sex differences in risk propensity, we used a functional network analysis based on RSFC. Functional connectivity density mapping (FCDM) is a recently proposed voxel-wise and model-free approach to the measurement of RSFC (Tomasi and Volkow, 2010, 2012b,d). It can be used to identify brain regions correlated with the measure of interest as well as to reveal details of functional networks centered on these regions when combined with seedbased RSFC (Tomasi and Volkow, 2012a). Accordingly, in this large-sample resting-state fMRI study, we developed a composite measure of the trait of general risk propensity (GRP) and examined its neural correlates in men and women using a whole brain search strategy based on FCDM combined with seed-based RSFC (Tomasi and Volkow, 2012a). We hypothesized that the neural correlates of GRP in men possibly differ from that in women in the resting-state functional networks centered at the regions previously implicated in sex-related differences in risktaking behaviors (Bolla et al., 2004; Lee et al., 2009), consistent with the view that RSFC reflects intrinsic representations of the brain's functional repertoire (Smith et al., 2009). In addition, we predicted that men and women may share the same neural correlates of GRP in resting-state functional networks centered at the regions supporting cognitive control, such as inferior frontal cortex (Aron et al., 2004), anterior insula or anterior cingulate cortex (Dosenbach et al., 2007, 2008), as indicated in previous studies regarding the RSFC of risk propensity (Cox et al., 2010; Han et al., 2012).

\section{MATERIALS AND METHODS PARTICIPANTS}

Three hundred and twenty-four healthy right-handed participants were recruited by advertisement for the present study. Five participants were excluded due to missing behavioral data. Three additional participants were excluded due to raw imaging data errors, and 12 participants were excluded due to bad raw imaging data after two board-certified radiologists, Yu and Qin, manually inspected the raw fMRI data for all of the participants. Furthermore, 15 participants were excluded due to excessive head motion during the fMRI scan (see the following section). Thus, there were 289 healthy participants in the final group, consisting of 131 men (mean age $=22.2$ years, $S D=2.5$ years) and 158 women (mean age $=23.2$ years, $S D=2.2$ years). All of the participants gave written informed consent, and this study was approved by the ethical committee of the Tianjin Medical University General Hospital.

\section{BEHAVIORAL PROCEDURES}

\section{Assessment of risk propensity}

A composite measure of risk propensity was developed from the following five scales, all of which are domain-free and stably measure different profiles of risk propensity. The scales used in this study were the Risk Propensity Scale (RPS), the Sensation Seeking Scale (SSS), the Eysenck Personality Questionnaire (EPQ), the Tridimensional Personality Questionnaire (TPQ), and the Barratt Impulsiveness Scale (BIS).

The RPS was developed to measure general risk-taking tendencies (Meertens and Lion, 2008). This is a short (7 items), practical, and easy-to-use scale that has been used in a number of previous experimental studies (Lion and Meertens, 2001, 2005).

The SSS Form V (Zuckerman et al., 1978) consists of 40 items related to four dimensions associated with sensation seeking: thrill and adventure seeking (SSS_TAS), the desire to engage in outdoor non-competitive sports or activities involving elements of risk; experience seeking (SSS_ES), the seeking of new experiences through the mind and senses and an unconventional style of life; disinhibition (SSS_DIS), particularly social disinhibition; and boredom susceptibility (SSS_BS), which is defined as the dislike of any repetition of experience, predictable, dull or boring people.

The EPQ (Eysenck, 1991) consists of 88 items that measure the personality traits: extraversion-introversion (EPQ_E), neuroticism or emotionality (EPQ_N), and psychotism (EPQ_P) or tough mindedness. And it also includes a lie subscale (EPQ_L). These constructs subsume elements of risk taking: impulsivity forms a part of the EPQ_P, and sensation seeking and venturesomeness form a part of the EPQ_E (Eysenck and Eysenck, 1985).

The TPQ (Cloninger et al., 1991) consists of 100 items that measure three dimensions of personality traits: novelty seeking (TPQ_NS), harm avoidance (TPQ_HA) and reward dependence (TPQ_RD). These traits have been previously used to predict risk taking behaviors, such as alcohol consumption, pathological gambling, substance abuse, and internet addiction (Kim and Grant, 2001; Hale et al., 2003; Skeel et al., 2008; Ko et al., 2010). Generally, persons with high novelty seeking, low harm avoidance, and low reward dependence are more likely to engage in these risky behaviors.

The BIS-11 (Patton et al., 1995) is a widely used self-reported measure of impulsive personality traits composed of three subscales: attentional (BIS_ATT), motor (BIS_MOT), and nonplanning (BIS_NP) impulsiveness. Similar to sensation seeking, impulsiveness has been demonstrated as being driving forces of risk taking (Harrison et al., 2005). These traits have also been linked to both risk taking behaviors measured by experimental tasks (Lee et al., 2008; Cheng and Lee, 2012) and risk-taking behaviors in real life, such as risky driving behavior (Cheng and Lee, 2012).

\section{Extraction of general risk propensity (GRP)}

Because each of the five scales measures risky propensity in different ways, principle component analysis (PCA) was used to extract an index reflecting GRP. PCA is a general technique used to extract common or shared variance from a set of measures 
(Costello and Osborne, 2005) and has been previously used in similar contexts (Need et al., 2009).

The data were analyzed using SPSS v17.0. The item scores from the RPS and scores on the relevant subscales of the SSS, EPQ, $\mathrm{TPQ}$, and BIS were used as variables. A total of 15 variables were entered into PCA with varimax rotation. The regression method was used to create the participant scores for each principal component. The score of the first rotated principal component was used as a measure of GRP in further analyses.

\section{MRI PROCEDURE}

\section{MR image acquisition}

MR images were acquired on a 3.0 Tesla MR scanner (General Electric, Milwaukee, WI, USA). During scanning, foam paddings and earplugs were used to limit head motion and to reduce scanning noise. All of the participants received a threedimensional magnetization prepared rapid acquisition gradient echo (3DMPRAGE) sequence (repetition time (TR)/echo time $(T E)=8.06 / 3.12 \mathrm{~ms}$, flip angle $=13^{\circ}, 176$ sagittal slices, voxel size $=1 \mathrm{~mm} \times 1 \mathrm{~mm} \times 1 \mathrm{~mm}$ ). The resting-state functional images were acquired using an echo-planar imaging (EPI) sequence that is sensitive to BOLD contrast with the following parameters: 40 slices, $T R / T E=2000 / 30 \mathrm{~ms}$, thickness/gap $=$ $4.0 / 0.0 \mathrm{~mm}$, voxel size $=3.75 \mathrm{~mm} \times 3.75 \mathrm{~mm} \times 4.0 \mathrm{~mm}$, FOV $=240 \times 240 \mathrm{~mm}$, matrix $=64 \times 64$, flip angle $=90^{\circ}$. Each functional run lasted for $6 \mathrm{~min}$ and contained 180 volumes. During the fMRI scans, all of the participants were instructed to keep their eyes closed, relax their minds, and move as little as possible.

\section{Imaging data preprocessing and analyses}

Data preprocessing. After inspection of the quality of the raw functional images, data preprocessing was performed using FSL tools (www.fmrib.ox.ac.uk/fsl) and AFNI (http://afni.nimh.nih. gov/afni). The following preprocessing steps were performed: discarding the first 10 slices for the magnetization equilibrium; slice timing correction; head motion correction; non-brain removal; temporal filtering $(0.01-0.1 \mathrm{~Hz})$ and regressing nuisance signals (6 motion parameters). Registration of the resting-state data to high-resolution T1-weighted images and the $\mathrm{T} 1$ non-linear data to a $3 \mathrm{~mm}$ isotropic MNI-152 standard space template (Montreal Neurological Institute) were performed. The resulting transformation matrices were combined to obtain a native MNI space transformation matrix and its inverse. Participants who had a maximum displacement greater than $2 \mathrm{~mm}$ in any of the cardinal directions $(x, y, z)$, and a maximum spin $(x, y, z)$ greater than $2^{\circ}$ were excluded from subsequent analysis.

Short- and long-range FCD. FCDM was used to characterize individual functional connectivity maps with high spatial resolution ( $\geq 3 \mathrm{~mm}$ isotropic) (Tomasi and Volkow, 2010). This method is free from the constraints of a priori selection of specific seed regions and allows the identification and location of functional hubs in a whole brain network from the perspectives of shortand long-range FCD (Tomasi and Volkow, 2012b). In the application of this method, a global FCD was first computed. On the basis of previous work, two voxels were considered functionally connected if their Pearson correlation coefficient was greater than 0.6 (Tomasi and Volkow, 2012b). The global FCD at a given voxel was defined as the number of functional connections between this voxel and all other voxels in the brain. Next, we used a "growing" algorithm developed in $\mathrm{C}$ to derive the short-range FCD. In this algorithm, for a given voxel, $x_{0}$, an additional voxel, $x_{j}$ was added to the list of neighbors of $x_{0}$ if it was adjacent to a voxel that was linked to $x_{0}$ by a continuous path of functionally connected voxels and the correlation coefficient between $x_{0}$ and $x_{j}$ was greater than 0.6. This calculation was repeated for all of the voxels that were adjacent to the neighbors of $x_{0}$ in an iterative manner until no new neighbors could be added to the list. The short-range FCD of $x_{0}$ was defined as the number of elements in the list of neighbors. The strength of the long-range FCD was equal to the difference between the global FCD and short-range FCD. These calculations were performed on all of the voxels in the brain. Thus, the strength of the short-range FCD in a voxel reflects the functional correlation between this voxel and other voxels within a local cluster, and the strength of the long-range FCD reflects the functional correlation between this voxel and other voxels located at a distance. Finally, the short- and long-range FCD maps were spatially smoothed using a Gaussian kernel of full-width at half-maximum (FWHM) of $8 \mathrm{~mm}$. For standardization purposes, the short- and long-range FCD of each voxel was divided by the global mean short- and long-range FCD, respectively.

RSFC networks. To reveal the specific networks associated with the GRP, the regions selected on the basis of the results of the short- and long-range FCD analyses were used as seed regions for the seed-based RSFC. The mean time series of each seed region were acquired by averaging the time series of all of the voxels within that region. Pearson's correlation coefficients were then computed between the mean time series of the seed region and the time series for each voxel in the brain. The correlation coefficients were then converted into $z$-values using Fisher's r-to-z transformation to improve their normality. Finally, an individual RSFC map was obtained.

\section{STATISTICAL ANALYSES}

Using SPM8, the normalized short- and long-range FCD maps computed for each participant were regressed on the variables of GRP, sex, and the GRP by sex interaction. The statistical significance was determined by Monte Carlo simulations to obtain a $p$-corrected $<0.05$ after correcting for whole brain comparisons. The corrected threshold corresponds to $p$-uncorrected $<0.001$ with a minimum cluster size of $513 \mathrm{~mm}^{3}$ (a gray mask with 55,342 voxels was used).

To assess the significance of the findings in a cluster of voxels rather than in a single voxel, the average strengths of the normalized short- and long-range FCD in regions with significant main or interaction effects were extracted from individual maps. These measures were then analyzed using SPSS v17.0. If an interaction effect was statistically significant, then post-hoc testing was performed using MODPROBE, which is an aid used to probe single-degree-of-freedom interactions in OLS and logistic regression analyses (Hayes and Matthes, 2009). 
Similar statistical analyses were applied to the RSFC maps. In this study, the RSFC map computed for each participant was regressed on the GRP, sex and sex by GRP. The whole brain comparisons were constrained by a mask composed of a set of regions showing significant connectivities with the seed region. The statistical significance was determined by Monte Carlo simulations to obtain the $p$-corrected $<0.05$. The corrected threshold corresponds to a $p$-uncorrected $<0.005$ with a minimum cluster size of $999 \mathrm{~mm}^{3}$ (which is dependent on the size of each mask).

\section{RESULTS \\ BEHAVIORAL MEASURE OF THE GRP}

The 15 measures were used to assess the GRP (for the mean and standard deviation of each measure, please see Table A1 in the Appendix). The variables correlated moderately well with each other, with no particularly large correlations (the largest absolute value of the correlation coefficient was 0.63 ). The KaiserMeyer-Olkin value was 0.748 , and the Bartlett's test results were significant (approx. Chi-Square $=1179.2, p<0.001$ ), indicating that PCA was appropriate. This revealed four components with eigenvalues greater than 1 . The loadings of each variable for each component after the rotation are shown in Table 1. Component 1 accounted for the most variance among the four components (21.8, 18.0, 8.7, and $7.3 \%$, respectively). The variables that loaded strongly on to Component 1 were those that were related to general risk-taking tendency, novelty/sensation seeking, venturesomeness and harm avoidance. For this reason, we identified Component 1 as reflecting GRP. High scores of GRP indicated risk taking and lower GRP scores indicated risk aversion.

Independent sample $t$-tests revealed that men had significantly higher GRP scores compared to women (mean/SD for men: 0.37/0.92; mean/SD for women: $-0.31 / 0.95 ; p<0.001)$.

\section{SHORT- AND LONG-RANGE FUNCTIONAL CONNECTIVITY}

The spatial distribution of the short-range FCD was highly localized in the posterior cingulate/ventral precuneus, occipital (cuneus and calcarine cortex) regions, middle cingulate cortex, anterior cingulate cortex/medial prefrontal cortices, the inferior parietal regions, thalamus and striatum. This distribution was similar to those previously reported by Tomasi and Volkow (2010, 2012d). The spatial distribution of the long-range FCD was also localized in the abovementioned regions with the maxima in the posterior cingulate/ventral precuneus and medial prefrontal cortices. Bilateral dorsolateral prefrontal, insula, lateral parietal regions and temporal cortices also had high long-range FCDs (Figure A1 in Appendix).

\section{General risk propensity}

The long-range FCD in the right inferior frontal gyrus (IFG) decreased with the GRP score $(p<0.05$, corrected) (Figure 1A, Table 2). The short-range FCD in the bilateral inferior frontal gyri and right dorsolateral prefrontal cortex also decreased with the GRP score, while that in the left fusiform gyri extending to the lingual gyri increased with the GRP score (Figure 1B, Table 2).

\section{Sex}

Strong sex differences for short- and long-range FCDs were found. Compared to men, women showed higher levels of
Table 1 | Loadings for principal components using varimax rotation.

\begin{tabular}{|c|c|c|c|c|}
\hline \multirow{2}{*}{$\begin{array}{l}\text { Roation } \\
\text { variables }\end{array}$} & \multicolumn{4}{|c|}{ Component } \\
\hline & $\begin{array}{c}1 \text { (Risk- } \\
\text { seeking) }\end{array}$ & 2 (Impulsivity) & $\begin{array}{c}3 \text { (Lack of } \\
\text { control) }\end{array}$ & $\begin{array}{c}4 \text { (Reward- } \\
\text { dependence) }\end{array}$ \\
\hline TPQ_HA & -0.774 & & & \\
\hline EPQ_E & 0.662 & & & 0.543 \\
\hline SSS_ES & 0.599 & & & \\
\hline EPQ_N & -0.578 & & 0.546 & \\
\hline SSS_DIS & 0.531 & & & \\
\hline RPS & 0.487 & & & \\
\hline SSS_TAS & 0.486 & & & \\
\hline BIS_MOT & & 0.814 & & \\
\hline TPQ_NS & 0.354 & 0.657 & & \\
\hline BIS_NP & & 0.652 & & -0.319 \\
\hline BIS_ATT & & 0.525 & 0.421 & \\
\hline EPQ_P & & & 0.699 & -0.320 \\
\hline SSS_BS & & & 0.670 & \\
\hline EPQ_L & & & -0.596 & \\
\hline TPQ_RD & & & & 0.824 \\
\hline
\end{tabular}

Abbreviations: BIS_ATT, attentional factors of the Barratt Impulsiveness Scale; BIS_MOT, motor factors of the Barratt Impulsiveness Scale; BIS_NP, nonplanning impulsiveness factors of the Barratt Impulsiveness Scale; EPQ_E, extraversion-introversion subscale of the Eysenck Personality Questionnaire; $E P Q_{-} L$, lie subscale; EPQ_N, neuroticism subscale of the Eysenck Personality Questionnaire; EPQ_P, psychotism subscale of the Eysenck Personality Questionnaire; RPS, Risk Propensity Scale; SSS_TAS, thrill and adventure seeking dimention of the Sensation Seeking Scale; SSS_ES, experience seeking dimention of the Sensation Seeking Scale; SSS_DIS, disinhibition dimention of the Sensation Seeking Scale; SSS_BS, boredom susceptibility dimention of the Sensation Seeking Scale; TPQ_NS, novelty seeking subscale of the Tridimensional Personality Questionnaire; TPQ_HA, harm avoidance subscale of the Tridimensional Personality Questionnaire; TPQ_RD, reward dependence subscale of the Tridimensional Personality Questionnaire.

long-range FCDs in the default mode network region (posterior cingulate cortex, medial frontal/orbitofrontal cortex, bilateral parahippocampal gyrus and its adjacent medial temporal cortices) (Greicius et al., 2003; Fox et al., 2005; Buckner et al., 2008), calcarine, superior frontal gyrus, cerebellum and pons, and showed lower levels of long-range FCDs in the bilateral ventral frontal and lateral orbitofrontal cortices, the bilateral insula, the right anterior/middle cingulate cortex and the left superior parietal lobe. The sex differences in short-range FCDs were similar, but more localized and weaker than the differences found in long-range FCDs (Figure A2 in Appendix).

\section{GRP by sex interaction}

The GRP by sex interaction was significant for long-range FCDs in the left inferior OFC and right supramarginal gyrus/postcentral gyrus (SII). Independent analyses of the average FCD values in the two regions validated the interaction effects [for the left inferior OFC $(\beta=0.25, p<0.001)$, for the right SII $(\beta=-0.25, p<0.001)]$. Post-hoc tests of these effects showed that the long-range FCD in the left inferior OFC was positively correlated with the GRP score for men $(\beta=0.11$, 


\section{A Long-range FCD}

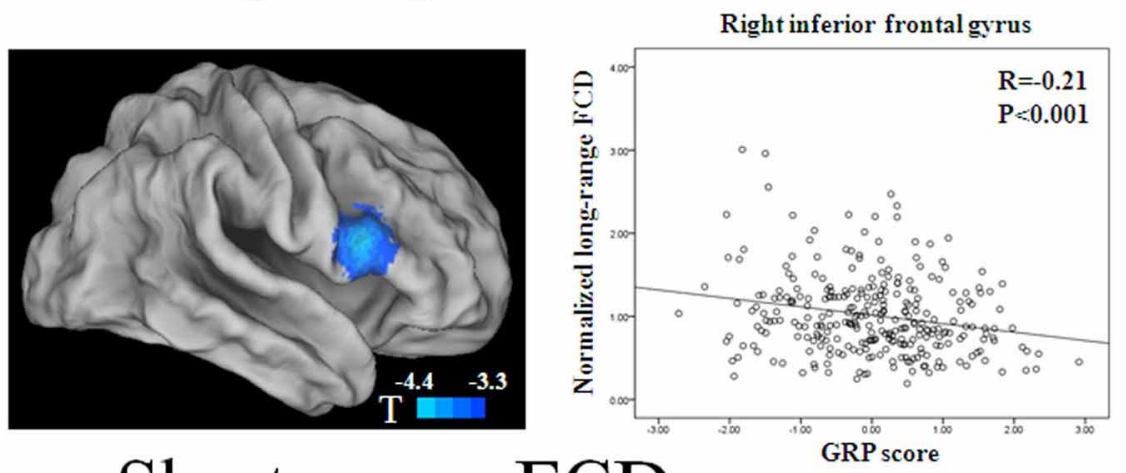

в Short-range FCD
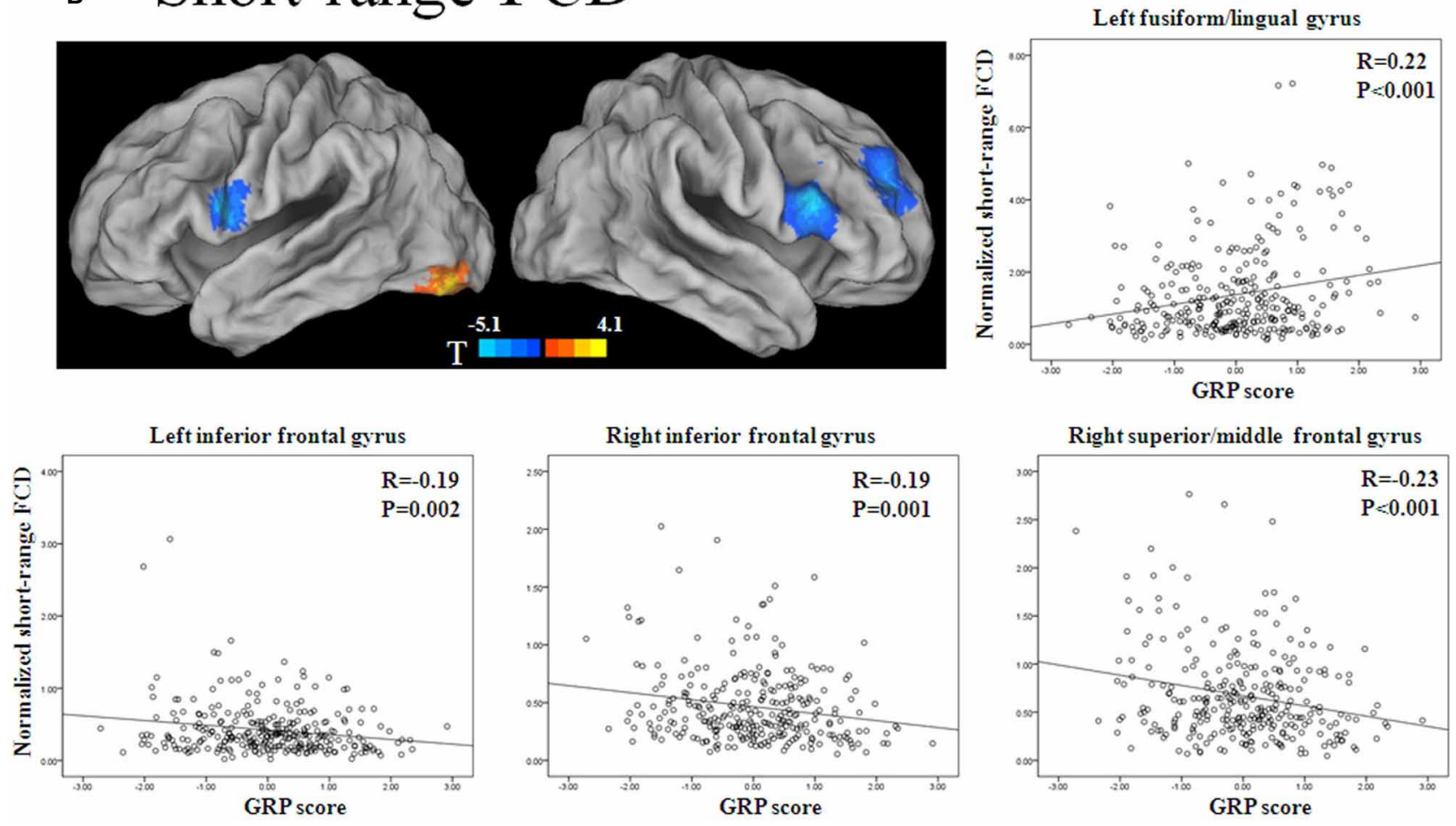

FIGURE 1 | Statistical significance ( $T$-score) of the correlation with the GRP score for long-range FCD (A) and short-range FCD (B) across 289 healthy participants, overlaid on the surface of the Colin template. The cool color represents a negative correlation, and the warm color represents a positive correlation. The images were created using the Computerized
Anatomical Reconstruction and Editing Toolkit (CARET) 5.62, which is a free software that was developed at Washington University (http://brainvis.wustl.edu/wiki/index.php/Caret:About). Scatter plots show GRP-related changes in long- or short-range FCD. The lines are linear fits of the data.
$S E=0.03, t=3.27, p=0.0012)$, but negatively correlated with the GRP score for women $(\beta=-0.08, S E=0.03, t=-2.72$, $p=0.007)$. Furthermore, the long-range FCD in the right SII was negatively correlated with the GRP score for men $(\beta=-0.27$, $S E=0.07, t=-4.05, p=0.0001)$, but was unrelated for women $(\beta=0.11, S E=0.06, t=1.87, p=0.06)$ (Figure 2, Table 3).

The GRP by sex interaction was also significant for shortrange FCDs in the left inferior OFC and right IFG. Independent analysis of the average FCD values in the two regions also validated the interaction effects [for the left inferior OFC ( $\beta=$ $0.22, p<0.001)$, for the right IFG $(\beta=-0.23, p<0.001)]$. Posthoc tests of these effects showed that the short-range FCD in the left inferior OFC was negatively correlated with the GRP score for women $(\beta=-0.07, S E=0.02, t=-4.07, p=0.0001)$, but not for men $(\beta=0.03, S E=0.02, t=1.59, p=0.1)$, and that the short-range FCD in the right IFG was negatively correlated with the GRP score for men $(\beta=-0.19, S E=0.04, t=-4.7$, 
Table 2 | Main effect of the general risk propensity scores on long- and short-range FCD.

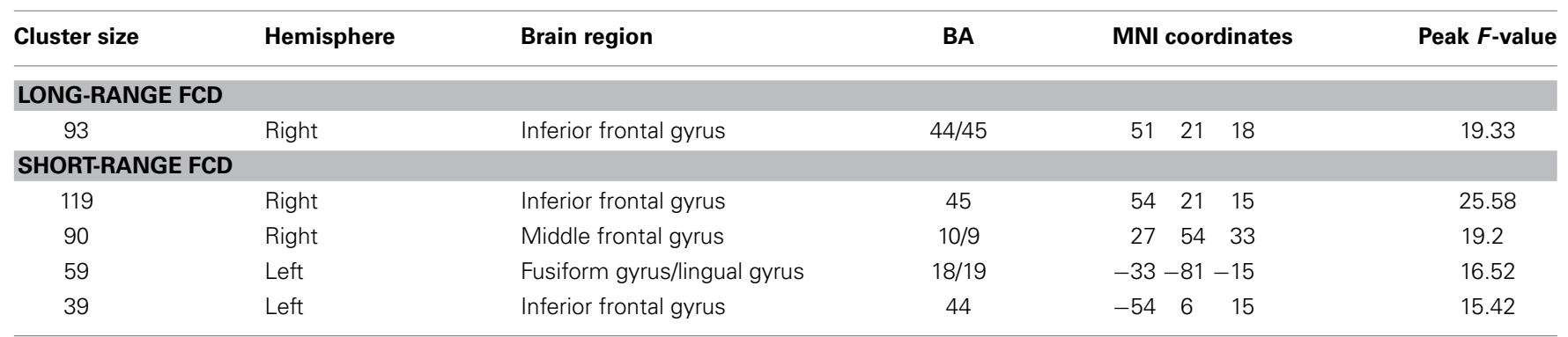

Abbreviations: BA, broadman area; FCD, functional connectivity density.

\section{A Long-range FCD}
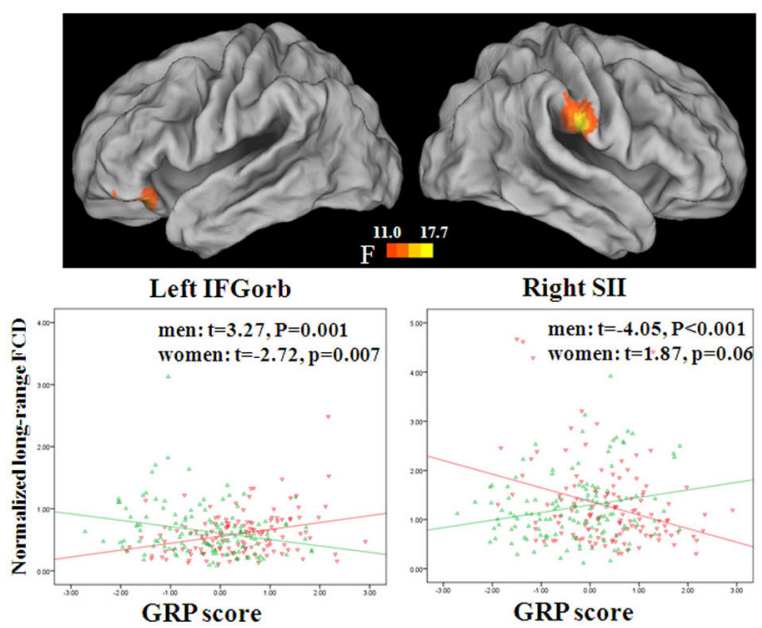

\section{Right SII}

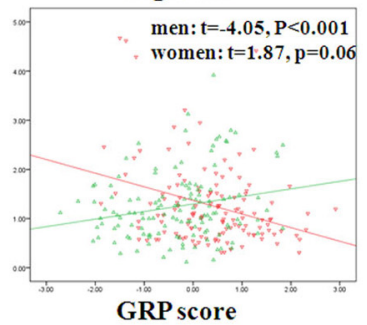

B Short-range FCD
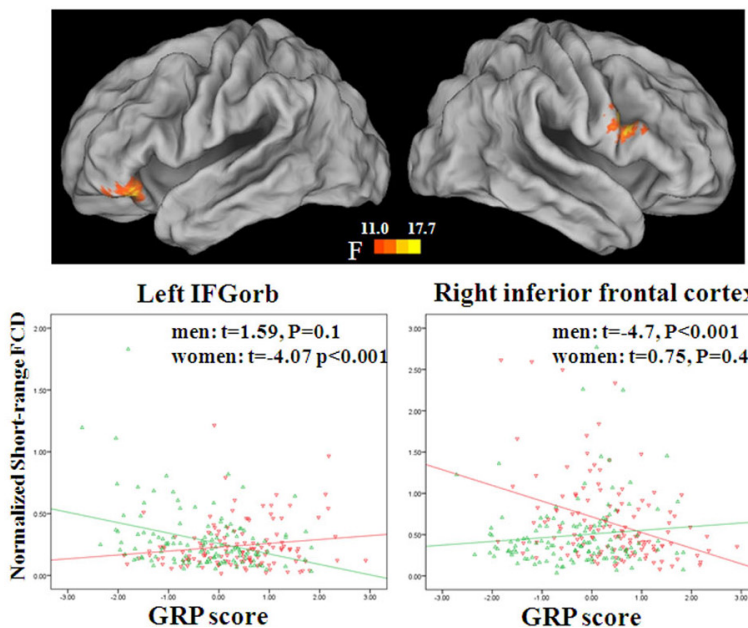

Right inferior frontal cortex

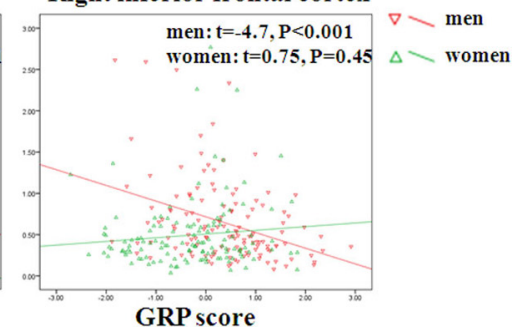

FIGURE 2 | Sex by GRP interaction effect for long-range FCD (A) and short-range FCD (B). The images were created using the CARET 5.62. Scatter plots show GRP-related changes in long- or short-range FCD in the male and female group. The lines are linear fits of the data.

$p<0.0001)$, but not for women $(\beta=0.03, S E=0.04, t=0.75$, $p=0.45)\left(\right.$ Figure 2, Table 3) ${ }^{1,2}$.

\section{RSFC NETWORKS}

Because both the short- and long-range FCD were correlated with GRP in the right IFG, we mapped the functional connectivity

\footnotetext{
${ }^{1}$ As one of the reviewers noticed, there were some extreme outliners in the functional density measures (Figure A3 in Appendix). When we re-analyzed the data after excluding these extreme outliers, we found that the GRP by sex interaction remained significant for long-range FCDs in the left inferior OFC $(\beta=0.22, p<0.001)$ and right SII $(\beta=-0.20, p=0.001)$. The GRP by sex interaction also remained significant for short-range FCDs in the left inferior OFC $(\beta=0.15, p=0.01)$ and right IFG $(\beta=-0.18, p=0.002)$.

${ }^{2}$ We acknowledge that the observed effect size is small, as one of the reviewers noticed. This is to be expected given that the low signal to noise ratio is inherent in resting state analyses. In order to test whether or not the interaction effect is robust, we randomly selected half of the original data set to determine whether or not the interaction effect remains significant. We found that the GRP by sex interaction remained significant for long-range FCDs in the left OFC $(\beta=0.27, p=0.002)$ and the right SII $(\beta=-0.33, p=0.000)$. The GRP by gender interaction was also significant for short-range FCDs in the left inferior OFC $(\beta=0.21, p=0.009)$ and right IFG $(\beta=-0.22, p=0.01)$.
}

network of this region. We found that the RSFCs between the right IFG and the right anterior insula and the bilateral precentral gyrus/inferior prefrontal gyrus, as well as the middle cingulated cortex (MCC) and its adjacent supplementary motor cortex (SMA) were negatively correlated with the GRP score $(p<0.05$, corrected) (Figure 3, Table 4).

The functional connectivity networks of the regions that showed a significant interaction effect in short- and long-range FCDs were also mapped. Significant interaction effects were found in the strength of the functional connectivities between the right SII and the bilateral insula extending to the striatum and thalamus, dorsal anterior cingulate cortex (dACC) and its adjacent medial prefrontal cortex, as well as the left cerebellar posterior lobe ( $p<0.05$, corrected). Post-hoc tests of the interaction effects showed that the strength of these functional connectivities was negatively correlated with the GRP for men (all $\beta \geq 0.04$, $S E \geq 0.01, t \leq-3.97, p<0.001)$, but was unrelated for women (all $\beta<0.02, S E \geq 0.01, t<1.1, p>0.05$ ) (Figure 4, Table 5). Significant interaction effects were also found in the strength of the functional connectivities between the left inferior OFC and adjacent cortex. Post-hoc tests of the interaction effect showed that the strength of this functional connectivity was positively 
Table 3 | Sex by the general risk propensity score interaction effects on long- and short-range FCD.

\begin{tabular}{|c|c|c|c|c|c|}
\hline Cluster size & Hemisphere & Brain region & BA & MNI coordinates & Peak $F$-value \\
\hline \multicolumn{6}{|c|}{ LONG-RANGE FCD } \\
\hline 68 & Right & Supramarginal gyrus/postcentral gyrus & $2 / 40$ & $66-18 \quad 24$ & 19.42 \\
\hline 30 & Left & Inferior orbitofrontal cortex & 11 & $-36 \quad 36-15$ & 18.96 \\
\hline \multicolumn{6}{|c|}{ SHORT-RANGE FCD } \\
\hline 27 & Right & Inferior frontal gyrus & $44 / 45$ & $\begin{array}{lll}57 & 12 & 24\end{array}$ & 17.6 \\
\hline 22 & Left & Inferior orbitofrontal cortex & 11 & $-42 \quad 36-18$ & 17.66 \\
\hline
\end{tabular}

Abbreviations: BA, broadman area; FCD, functional connectivity density.

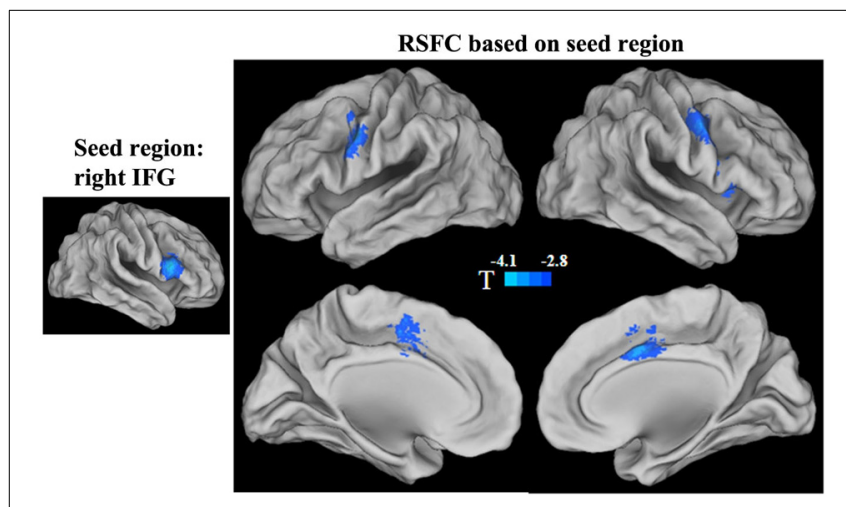

FIGURE 3 | Statistical significance ( $T$-score) of the correlation with the GRP score for the strength of functional connectivities in the right IFG seed region. The cool color represents a negative correlation.

correlated with the GRP for men $(\beta=0.03, S E=0.01, t=2.64$, $p=0.009)$, but was negatively correlated for women $(\beta=-0.03$, $S E=0.01, t=-3.03, p=0.003)$.

\section{DISCUSSION}

Previous studies have emphasized the utility of RSFC methods in the investigation of inter-individual differences in brain function associated with enduring behavioral traits and tendencies (Di Martino et al., 2009; Cox et al., 2010; Hoptman et al., 2010; Adelstein et al., 2011). In the present study, we examined the neural correlates of the trait of GRP in resting-state fMRI in men and women using the recently developed FCDM method combined with seed-based RSFC. Our results revealed the existence of both shared and distinct functional networks associated with risk propensity in men and women.

\section{RSFC RELIABLY MEASURES INTRINSIC FUNCTIONAL ARCHITECTURE AND SEX DIFFERENCES}

In the current study, using FCDM, we identified the posterior cingulate/ventral precuneus and medial prefrontal cortices as the core regions in the intrinsic functional architecture, consistent with previous studies (Buckner et al., 2009; Tomasi and Volkow, 2010, 2012b; Zuo et al., 2012). We also found a significant sex difference for both short- and long-range FCDs. Women showed higher levels of short- and long-range FCDs in the default mode network regions, and lower levels of short- and long-range FCDs in the ventral frontal cortex and insula. This result is consistent with previous findings that the organization of intrinsic brain activity differs between males and females (Biswal et al., 2010; Zuo et al., 2010; Tomasi and Volkow, 2012b,c,d; Zuo et al., 2012; Filippi et al., 2013). The similarity of these results suggests that resting-state functional network analysis can provide a reliable measure for the neural correlates of the trait of GRP and its sex differences.

\section{SEX DIFFERENCES IN THE NEURAL CORRELATES OF GENERAL RISK PROPENSITY}

In the current study, the interaction between sex and GRP was found in short- and/or long-range FCDs in the right secondary somatosensory cortex (SII) and the left OFC, suggesting that the resting-state neural correlates of risk propensity may differ between men and women. Seed-based RSFC further revealed the detailed functional connectivities associated with sex differences in the GRP.

A local network centered at the left inferior OFC was found to be differentially correlated with GRP in men and in women. This finding is consistent with previous task-based fMRI studies in which the inferior lateral OFC has also been identified as contributing to sex-related differences in risk-taking behavior (Bolla et al., 2004; Lee et al., 2009). In addition, we found that women have higher spontaneous activities within a local network centered at the left lateral OFC coupled with lower risk propensity. This is consistent with Lee et al's study, in which activity in the lateral OFC has been found to correlate negatively with the rate of selecting risky choices in women (Lee et al., 2009). However, these previous studies focused on the differences in the task-induced activity of the lateral OFC between men and women. The current study based on resting-state functional connectivity suggests that the functional connectivities within the lateral OFC also contributed to sex differences in GRP. Therefore, the results of the current study lend further support to a sex-related modulation of neural activity in the lateral OFC and extend this effect to spontaneous neural activity during rest.

A resting-state network centered at the right SII was also differentially associated with GRP in men and in women. This network included the bilateral insula extending to the striatum and thalamus, dACC, and left cerebellum. Increasing anatomical evidence has shown that extensive connections exist between the SII and thalamus (Krubitzer and Kaas, 1992; Qi et al., 2002), between the thalamus, dorsal anterior insula and dACC (Mufson and Mesulam, 1984; Vogt et al., 1987), and between the SII and posterior insula (Mesulam and Mufson, 1982; Mufson and Mesulam, 
Table 4 | Main effect of the general risk propensity scores on the RSFC in the right IFG seed region.

\begin{tabular}{|c|c|c|c|c|c|c|c|}
\hline \multirow{2}{*}{$\begin{array}{c}\text { Cluster size } \\
50\end{array}$} & \multirow{2}{*}{$\begin{array}{l}\text { Hemisphere } \\
\text { Right }\end{array}$} & \multirow{2}{*}{$\begin{array}{l}\text { Brain region } \\
\text { Anterior insular }\end{array}$} & \multirow{2}{*}{$\begin{array}{c}\text { BA } \\
13 / 45\end{array}$} & \multicolumn{3}{|c|}{ MNI coordinates } & \multirow{2}{*}{$\begin{array}{c}\text { Peak } \boldsymbol{F} \text {-value } \\
16.57\end{array}$} \\
\hline & & & & 45 & 21 & 6 & \\
\hline 46 & Left & Precentral gyrus/IFG & $6 / 9$ & -57 & 3 & 36 & 13.52 \\
\hline 62 & Right & Precentral gyrus/IFG & $6 / 9$ & 51 & 3 & 30 & 11.14 \\
\hline 57 & Bilateral & MCC/SMA & $32 / 24 / 6$ & 3 & 3 & 45 & 10.8 \\
\hline
\end{tabular}

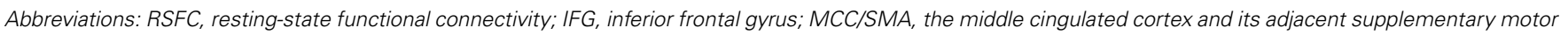
cortex.

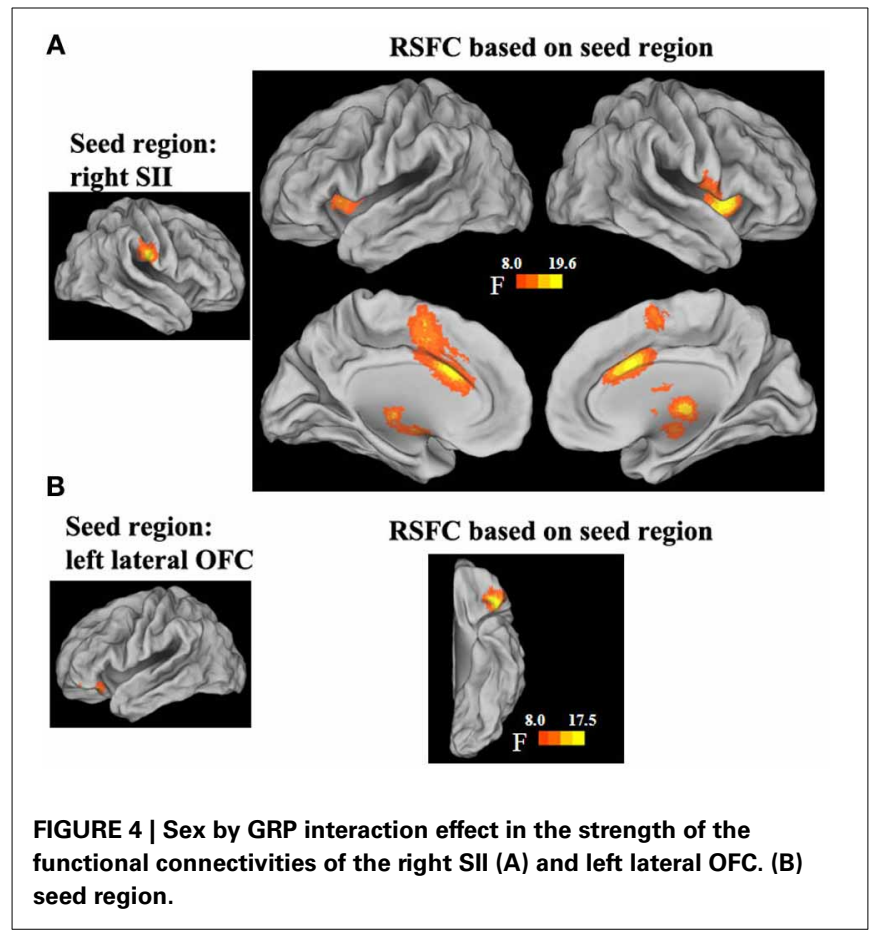

1982). These anatomical connections may be the structural basis of the resting-state network centered at the right SII. Although the role of the SII cortex in risk processing remains unclear, most of the component regions in this functional network have been implicated in risk processing based on a quantitative coordinatebased meta-analysis of studies that investigated the neural representations of risk (Mohr et al., 2010). Specifically, the anterior insula and thalamus have been identified as being involved in emotional risk processing and the dACC has been identified as involved in cognitive risk processing (Mohr et al., 2010). Our finding that the resting-state functional connectivities between the SII and these regions are differentially associated with GRP in men and in women suggests that future task-based fMRI studies should focus on the role of the SII in risk processing.

It is noteworthy that the dorsal anterior insula and the dACC, together with some cortical and subcortical structures such as thalamus, constitute a functional network which has been suggested as being involved in detecting the salience of external and internal stimuli, whether cognitive, homeostatic, or emotional, and prompting appropriate behavioral responses (Dosenbach et al., 2007; Seeley et al., 2007; Menon and Uddin, 2010; Legrain et al.,
2011; Beissner et al., 2013). The unique functions identified in this network point to the possibility that the differential processing of salience information may determine at least some sex differences in GRP. Future research may shed some light on the nature of this possible connection.

\section{SHARED NEURAL CORRELATES OF GENERAL RISK PROPENSITY IN BOTH MEN AND WOMEN}

For both men and women, GRP was linked with short- and long-range FCD in the right IFG, indicating that the stronger the resting-state functional connectivity of the right IFG, the lower the risk propensity. Task-induced response studies have shown that activity in the right IFG is related to risk aversion (Christopoulos et al., 2009; D'Acremont et al., 2009), consistent with lesion (Clark et al., 2003) and resting EEG (Gianotti et al., 2009) studies. Our results are consistent with these studies. Using seed-based RSFC, we further found a negative correlation between GRP and the strength of functional connectivity between the right IFG and right anterior insula. This is consistent with the results of a previous resting-state fMRI study in which risk-aversion was associated with stronger positive functional connectivity between the right IFG and the right insula (Cox et al., 2010). Our results not only replicate this previous finding but also reveal that the strength of the functional connectivity between the right IFG and MCC covaries with GRP. The MCC, which is located close to the dACC where we found sex differences in GRP, is also named as dACC in some studies (such as Dosenbach et al., 2007; Seeley et al., 2007; Menon and Uddin, 2010). Considering that both the MCC and right anterior insula are the core regions in a functional network (Dosenbach et al., 2007; Seeley et al., 2007; Menon and Uddin, 2010; Legrain et al., 2011; Beissner et al., 2013), these two regions may play similar roles in risk propensity.

We also found that short-range FCDs in the right dorsolateral prefrontal cortex, in addition to bilateral inferior frontal gyri and adjacent anterior insula, were negatively correlated with GRP. The dorsolateral prefrontal cortex is a core region in the frontoparietal component of the control system in the resting brain (Dosenbach et al., 2007, 2008). Previous studies have supported its role in risky decision making: transient suppression of activity in the right dorsolateral prefrontal cortex increases risk-taking behavior (Knoch et al., 2006), while upregulation of activity in this region decreases risk-taking behavior (Fecteau et al., 2007a,b). Our finding is consistent with these previous studies, and suggests that the functional connectivities within the dorsolateral prefrontal cortex are also related to GRP in both men and women. 
Table 5 | Sex by the general risk propensity score interaction effects on the RSFC in the right SII seed region.

\begin{tabular}{|c|c|c|c|c|c|}
\hline Cluster size & Hemisphere & Brain region & BA & MNI coordinates & Peak $F$-value \\
\hline 750 & Right & Insula/striatum/thalamus & 13 & $12-3$ & 19.63 \\
\hline 300 & Bilateral & $\begin{array}{l}\text { Dorsal anterior cingulate cortex/ } \\
\text { medial prefrontal cortex }\end{array}$ & $32 / 24 / 6$ & $18 \quad 33$ & 18.07 \\
\hline 251 & Left & Insula/striatum/thalamus & 13 & $-30 \quad 150$ & 15.03 \\
\hline 144 & Left & Cerebellum posterior lobe & & $-30-45-45$ & 14.22 \\
\hline
\end{tabular}

Abbreviations: RSFC, resting-state functional connectivity; SII, supramarginal gyrus/postcentral gyrus.

\section{SEVERAL ISSUES NEED TO BE ADDRESSED}

The present study is based on the development of a composite measure of the trait of GRP. Generally there are two different views on measuring risk propensity (Harrison et al., 2005). On one view, risk taking is a function of a decision maker's risk perception and willingness to assume these perceived risks for an expected return. Choices across different risk domains therefore depend on enduring individual differences in risk perception and preferences. The alternative view is that risk propensity is an unstable trait across decision risk domains (such as finance, health/safety, social and so on) (such as Weber et al., 2002). Accordingly, there are two approaches to measure risk propensity. One approach, used here, is to identify relatively stable and domain-free traits associated with risk taking, with the traits of sensation seeking and impulsivity seen as particularly important (Harrison et al., 2005). The other approach is to measure separate aspects of risk propensity across multiple domains. In the present study, we have assumed that risk propensity, measured by the first method, is a stable individual trait and likely to be reflected in differences in resting-state brain functional activity. This is supported by the fact that our findings on the shared neural correlates of GRP in both men and women are consistent with a previous RSFC study (Cox et al., 2010), which similarly used healthy adults as participants.

In the present study, we used a whole brain search strategy based on FCDM combined with seed-based RSFC to examine the neural correlates of the GRP in men and women. One of the merits of this method is that it can identify the functional networks underlying risk propensity and its sex differences. This is an advance compared to the previous task-based fMRI studies (Bolla et al., 2004; Lee et al., 2009), which focused on activation of individual regions during risk-taking tasks. Because an understanding of how the human brain produces cognition and complex behaviors ultimately depends on the knowledge of a large scale brain organization (Bressler and Menon, 2010), the current findings improve our understanding of the neural basis of sex differences in risk propensity. Although it is difficult to reach a firm conclusion regarding the specific functional role of each region or network found by resting-state fMRI per se due to the lack of a directly involved task, the correspondence between regions identified by the two methods suggests some form of connection (Smith et al., 2009). Following a Hebbian view of experience dependent synaptic enhancement (Hebb, 1949), the correspondence between the regions identified by the current resting-state study and previous task-based studies suggests that the differences and similarities between men and women in the neural correlates of
GRP may, at least in part, reflect a longstanding history of coactivation of specific brain regions as a network involved in the differential processing of risk in men and women. However, the exact nature of this connection awaits further research.

\section{CONCLUSIONS}

The present study investigated the relationship between the enduring trait of GRP and intrinsic functional connectivity networks in men and women. We found patterns of sex differences that may underlie potential qualitative differences in the processing of risk by men and women. This work provides a new perspective on the brain-behavioral relationships in risky decision making and contributes to our understanding of sex differences in risk propensity.

\section{AUTHOR CONTRIBUTIONS}

All authors were involved in the design and implementation of the study and the writing of the manuscript. Authors Yuan Zhou, Shu $\mathrm{Li}$, Chunshui Yu, and Tianzi Jiang devised the concept and supervised the study. Authors Huandong Li, Wen Qin, and Maohu Zhu collected the imaging data. Authors Yuan Zhou, Huandong Li, Maohu Zhu, and Li-Lin Rao carried out the analysis. Authors Shu Li, John Dunn, Chunshui Yu, and Tianzi Jiang joined in the interpretation of data.

\section{ACKNOWLEDGMENTS}

This research was partially supported by the National Basic Research Program of China (973 Program, Nos. 2011CB707800 and 2011CB711000), National Natural Science Foundation of China (Nos. 30900487, 31170976 and 91132301), Chinese Academy of Sciences Visiting Professorship for Senior International Scientists and Project for Young Scientists Fund of the Institute of Psychology, Chinese Academy of Sciences (Y0CX163S01). The authors thank Dr. Kun Zhou for her help in collecting data and Dr. Yuqing Zhang, Dr. Zhuyuan Liang, Dr. Rui Zheng, and Dr. Xiaotian Wang for their technical assistance and comments on this study.

\section{REFERENCES}

Adelstein, J. S., Shehzad, Z., Mennes, M., Deyoung, C. G., Zuo, X. N., Kelly, C., et al. (2011). Personality is reflected in the brain's intrinsic functional architecture. PLoS ONE 6:e27633. doi: 10.1371/journal.pone.0027633

Aron, A. R., Robbins, T. W., and Poldrack, R. A. (2004). Inhibition and the right inferior frontal cortex. Trends Cogn. Sci. 8, 170-177. doi: 10.1016/j.tics.2004.02.010

Beissner, F., Meissner, K., Bar, K. J., and Napadow, V. (2013). The autonomic brain: an activation likelihood estimation meta-analysis for central 
processing of autonomic function. J. Neurosci. 33, 10503-10511. doi: 10.1523/JNEUROSCI.1103-13.2013

Biswal, B. B., Mennes, M., Zuo, X. N., Gohel, S., Kelly, C., Smith, S. M., et al. (2010). Toward discovery science of human brain function. Proc. Natl. Acad. Sci. U.S.A. 107, 4734-4739. doi: 10.1073/pnas.0911855107

Biswal, B., Yetkin, F. Z., Haughton, V. M., and Hyde, J. S. (1995). Functional connectivity in the motor cortex of resting human brain using echo-planar MRI. Magn. Reson. Med. 34, 537-541. doi: 10.1002/mrm.1910340409

Bolla, K. I., Eldreth, D. A., Matochik, J. A., and Cadet, J. L. (2004). Sex-related differences in a gambling task and its neurological correlates. Cereb. Cortex 14, 1226-1232. doi: 10.1093/cercor/bhh083

Bressler, S. L., and Menon, V. (2010). Large-scale brain networks in cognition: emerging methods and principles. Trends Cogn. Sci. 14, 277-290. doi: 10.1016/j.tics.2010.04.004

Buckner, R. L., Andrews-Hanna, J. R., and Schacter, D. L. (2008). The brain's default network: anatomy, function, and relevance to disease. Ann. N.Y. Acad. Sci. 1124, 1-38. doi: 10.1196/annals.1440.011

Buckner, R. L., Sepulcre, J., Talukdar, T., Krienen, F. M., Liu, H., Hedden, T. et al. (2009). Cortical hubs revealed by intrinsic functional connectivity: mapping, assessment of stability, and relation to Alzheimer's disease. J. Neurosci. 29, 1860-1873. doi: 10.1523/JNEUROSCI.5062-08.2009

Byrnes, J. P., Miller, D. C., and Schafer, W. D. (1999). Gender differences in risk taking: a meta-analysis. Psychol. Bull. 125, 367-383. doi: 10.1037/00332909.125.3.367

Cheng, A. S., and Lee, H. C. (2012). Risk-taking behavior and response inhibition of commuter motorcyclists with different levels of impulsivity. Trans. Res. Part F Traffic Psychol. Behav. 15, 535-543. doi: 10.1016/j.trf.2012.05.005

Christopoulos, G. I., Tobler, P. N., Bossaerts, P., Dolan, R. J., and Schultz, W. (2009). Neural correlates of value, risk, and risk aversion contributing to decision making under risk. J. Neurosci. 29, 12574-12583. doi: 10.1523/JNEUROSCI.261409.2009

Clark, L., Manes, F., Antoun, N., Sahakian, B. J., and Robbins, T. W. (2003). The contributions of lesion laterality and lesion volume to decision-making impairment following frontal lobe damage. Neuropsychologia 41, 1474-1483. doi: 10.1016/S0028-3932(03)00081-2

Cloninger, C. R., Przybeck, T. R., and Svrakic, D. M. (1991). The tridimensional personality questionnaire: U.S. normative data. Psychol. Rep. 69, 1047-1057.

Costello, A. B., and Osborne, J. W. (2005). Best practices in exploratory factor analysis: four recommendations for getting the most from your analysis. Pract. Assess. Res. Eval. 10, 1-9. Available online at: http://pareonline. net/pdf/v10n7.pdf.

Cox, C. L., Gotimer, K., Roy, A. K., Castellanos, F. X., Milham, M. P., and Kelly, C. (2010). Your resting brain CAREs about your risky behavior. PLoS ONE 5:e12296. doi: 10.1371/journal.pone.0012296

Croson, R., and Gneezy, U. (2009). Gender differences in preferences. J. Econ. Lit. 47, 448-474. doi: 10.1257/jel.47.2.448

D’Acremont, M., Lu, Z. L., Li, X., Van Der Linden, M., and Bechara, A. (2009). Neural correlates of risk prediction error during reinforcement learning in humans. Neuroimage 47, 1929-1939. doi: 10.1016/j.neuroimage.2009.04.096

Di Martino, A., Shehzad, Z., Kelly, C., Roy, A. K., Gee, D. G., Uddin, L. Q., et al. (2009). Relationship between cingulo-insular functional connectivity and autistic traits in neurotypical adults. Am. J. Psychiatry 166, 891-899. doi 10.1176/appi.ajp.2009.08121894

Dohmen, T., Falk, A., Huffman, D., Sunde, U., Schupp, J., and Wagner, G. G. (2011). Individual risk attitudes: measurement, determinants, and behavioral consequences. J. Eur. Econ. Assoc. 9, 522-550. doi: 10.1111/j.1542-4774.2011.01015.x

Dosenbach, N. U., Fair, D. A., Cohen, A. L., Schlaggar, B. L., and Petersen, S. E. (2008). A dual-networks architecture of top-down control. Trends Cogn. Sci. 12, 99-105. doi: 10.1016/j.tics.2008.01.001

Dosenbach, N. U., Fair, D. A., Miezin, F. M., Cohen, A. L., Wenger, K. K., Dosenbach, R. A., et al. (2007). Distinct brain networks for adaptive and stable task control in humans. Proc. Natl. Acad. Sci. U.S.A. 104, 11073-11078. doi: 10.1073/pnas.0704320104

Eysenck, H. J. (1991). Manual of the Eysenck Personality Scales (EPS Adult). London: Hodder and Stoughton.

Eysenck, H. J., and Eysenck, M. W. (1985). Personality and Individual Differences: A Natural Science Approach. New York, NY: Plenum Press. doi: 10.1007/978-14613-2413-3
Fecteau, S., Knoch, D., Fregni, F., Sultani, N., Boggio, P., and Pascual-Leone, A. (2007a). Diminishing risk-taking behavior by modulating activity in the prefrontal cortex: a direct current stimulation study. J. Neurosci. 27, 12500-12505. doi: 10.1523/JNEUROSCI.3283-07.2007

Fecteau, S., Pascual-Leone, A., Zald, D. H., Liguori, P., Theoret, H., Boggio, P. S., et al. (2007b). Activation of prefrontal cortex by transcranial direct current stimulation reduces appetite for risk during ambiguous decision making. J. Neurosci. 27, 6212-6218. doi: 10.1523/JNEUROSCI.0314-07.2007

Filippi, M., Valsasina, P., Misci, P., Falini, A., Comi, G., and Rocca, M. A. (2013). The organization of intrinsic brain activity differs between genders: a restingstate fMRI study in a large cohort of young healthy subjects. Hum. Brain Mapp. 34, 1330-1343. doi: 10.1002/hbm.21514

Fox, M. D., and Raichle, M. E. (2007). Spontaneous fluctuations in brain activity observed with functional magnetic resonance imaging. Nat. Rev. Neurosci. 8, 700-711. doi: 10.1038/nrn2201

Fox, M. D., Snyder, A. Z., Vincent, J. L., Corbetta, M., Van Essen, D. C., and Raichle, M. E. (2005). The human brain is intrinsically organized into dynamic, anticorrelated functional networks. Proc. Natl. Acad. Sci. U.S.A. 102, 9673-9678. doi: 10.1073/pnas.0504136102

Fox, M. D., Snyder, A. Z., Vincent, J. L., and Raichle, M. E. (2007). Intrinsic fluctuations within cortical systems account for intertrial variability in human behavior. Neuron 56, 171-184. doi: 10.1016/j.neuron.2007.08.023

Fox, M. D., Snyder, A. Z., Zacks, J. M., and Raichle, M. E. (2006). Coherent spontaneous activity accounts for trial-to-trial variability in human evoked brain responses. Nat. Neurosci. 9, 23-25. doi: 10.1038/nn1616

Gianotti, L. R., Knoch, D., Faber, P. L., Lehmann, D., Pascual-Marqui, R. D., Diezi, C., et al. (2009). Tonic activity level in the right prefrontal cortex predicts individuals' risk taking. Psychol. Sci. 20, 33-38. doi: 10.1111/j.14679280.2008.02260.x

Greicius, M. D., Krasnow, B., Reiss, A. L., and Menon, V. (2003). Functional connectivity in the resting brain: a network analysis of the default mode hypothesis. Proc. Natl. Acad. Sci. U.S.A. 100, 253-258. doi: 10.1073/pnas.0135058100

Hale, R. L., Whiteman, S., Muehl, K., and Faynberg, E. (2003). Tridimensional personality traits of college student marijuana users. Psychol. Rep. 92, 661-666. doi: 10.2466/pr0.2003.92.2.661

Han, S. D., Boyle, P. A., Arfanakis, K., Fleischman, D. A., Yu, L., Edmonds, E. C., et al. (2012). Neural intrinsic connectivity networks associated with risk aversion in old age. Behav. Brain Res. 227, 233-240. doi: 10.1016/j.bbr.2011. 10.026

Harrison, J. D., Young, J. M., Butow, P., Salkeld, G., and Solomon, M. J. (2005) Is it worth the risk? A systematic review of instruments that measure risk propensity for use in the health setting. Soc. Sci. Med. 60, 1385-1396. doi: 10.1016/j.socscimed.2004.07.006

Hayes, A. F., and Matthes, J. (2009). Computational procedures for probing interactions in OLS and logistic regression: SPSS and SAS implementations. Behav. Res. Methods 41, 924-936. doi: 10.3758/BRM.41.3.924

Hebb, D. O. (1949). The Organization of Behavior. New York, NY: Wiley.

Hoptman, M. J., D’angelo, D., Catalano, D., Mauro, C. J., Shehzad, Z. E., Kelly, A. M., et al. (2010). Amygdalofrontal functional disconnectivity and aggression in schizophrenia. Schizophr. Bull. 36, 1020-1028. doi: 10.1093/schbul/sbp012

Hsu, M., Bhatt, M., Adolphs, R., Tranel, D., and Camerer, C. F. (2005). Neural systems responding to degrees of uncertainty in human decision-making. Science 310, 1680-1683. doi: 10.1126/science.1115327

Kim, S. W., and Grant, J. E. (2001). Personality dimensions in pathological gambling disorder and obsessive-compulsive disorder. Psychiatry Res. 104, 205-212. doi: 10.1016/S0165-1781(01)00327-4

Knoch, D., Gianotti, L. R., Pascual-Leone, A., Treyer, V., Regard, M., Hohmann, M., et al. (2006). Disruption of right prefrontal cortex by low-frequency repetitive transcranial magnetic stimulation induces risktaking behavior. J. Neurosci. 26, 6469-6472. doi: 10.1523/JNEUROSCI.080406.2006

Ko, C. H., Hsiao, S., Liu, G. C., Yen, J. Y., Yang, M. J., and Yen, C. F. (2010) The characteristics of decision making, potential to take risks, and personality of college students with Internet addiction. Psychiatry Res. 175, 121-125. doi: 10.1016/j.psychres.2008.10.004

Krubitzer, L. A., and Kaas, J. H. (1992). The somatosensory thalamus of monkeys: cortical connections and a redefinition of nuclei in marmosets. J. Comp. Neurol. 319, 123-140. doi: 10.1002/cne.903190111 
Lee, T. M., Chan, C. C., Han, S. H., Leung, A. W., Fox, P. T., and Gao, J. H. (2008). An event-related fMRI study on risk taking by healthy individuals of high or low impulsiveness. Neurosci. Lett. 438, 138-141. doi: 10.1016/j.neulet.2008.04.061

Lee, T. M., Chan, C. C., Leung, A. W., Fox, P. T., and Gao, J. H. (2009). Sex-related differences in neural activity during risk taking: an fMRI study. Cereb. Cortex 19, 1303-1312. doi: 10.1093/cercor/bhn172

Legrain, V., Iannetti, G. D., Plaghki, L., and Mouraux, A. (2011). The pain matrix reloaded: a salience detection system for the body. Prog. Neurobiol. 93, 111-124. doi: 10.1016/j.pneurobio.2010.10.005

Lion, R., and Meertens, R. M. (2001). Seeking information about a risky medicine: effects of risk-taking tendency and accountability. J. Appl. Soc. Psychol. 31, 778-795. doi: 10.1111/j.1559-1816.2001.tb01413.x

Lion, R., and Meertens, R. M. (2005). Security or opportunity: the influence of risk-taking tendency on risk information preference. J. Risk Res. 8, 283-294. doi: 10.1080/1366987042000192435

Liu, X., Zhu, X. H., and Chen, W. (2011). Baseline BOLD correlation predicts individuals' stimulus-evoked BOLD responses. Neuroimage 54, 2278-2286. doi: 10.1016/j.neuroimage.2010.10.001

Meertens, R. M., and Lion, R. (2008). Measuring an individual's tendency to take risks: the risk propensity scale. J. Appl. Soc. Psychol. 38, 1506-1520. doi: 10.1111/j.1559-1816.2008.00357.x

Mennes, M., Kelly, C., Zuo, X. N., Di Martino, A., Biswal, B. B., Castellanos, F. X., et al. (2010). Inter-individual differences in resting-state functional connectivity predict task-induced BOLD activity. Neuroimage 50, 1690-1701. doi: 10.1016/j.neuroimage.2010.01.002

Mennes, M., Zuo, X. N., Kelly, C., Di Martino, A., Zang, Y. F., Biswal, B., et al. (2011). Linking inter-individual differences in neural activation and behavior to intrinsic brain dynamics. Neuroimage 54, 2950-2959. doi: 10.1016/j.neuroimage.2010.10.046

Menon, V., and Uddin, L. Q. (2010). Saliency, switching, attention and control: a network model of insula function. Brain Struct. Funct. 214, 655-667. doi: 10.1007/s00429-010-0262-0

Mesulam, M. M., and Mufson, E. J. (1982). Insula of the old world monkey. III: Efferent cortical output and comments on function. J. Comp. Neurol. 212, 38-52. doi: 10.1002/cne.902120104

Mohr, P. N., Biele, G., and Heekeren, H. R. (2010). Neural processing of risk. J. Neurosci. 30, 6613-6619. doi: 10.1523/JNEUROSCI.0003-10.2010

Mufson, E. J., and Mesulam, M. M. (1982). Insula of the old world monkey. II: Afferent cortical input and comments on the claustrum. J. Comp. Neurol. 212, 23-37. doi: 10.1002/cne.902120103

Mufson, E. J., and Mesulam, M. M. (1984). Thalamic connections of the insula in the rhesus monkey and comments on the paralimbic connectivity of the medial pulvinar nucleus. J. Comp. Neurol. 227, 109-120. doi: 10.1002/cne.902270112

Need, A. C., Attix, D. K., McEvoy, J. M., Cirulli, E. T., Linney, K. L., Hunt, P., et al. (2009). A genome-wide study of common SNPs and CNVs in cognitive performance in the CANTAB. Hum. Mol. Genet. 18, 4650-4661. doi: 10.1093/hmg/ddp413

Park, H. J., and Friston, K. (2013). Structural and functional brain networks: from connections to cognition. Science 342:1238411. doi: 10.1126/science.1238411

Patton, J. H., Stanford, M. S., and Barratt, E. S. (1995). Factor structure of the Barratt impulsiveness scale. J. Clin. Psychol. 51, 768-774. doi: 10.1002/10974679(199511)51:6<768::AID-JCLP2270510607>3.0.CO;2-1

Paulus, M. P., Rogalsky, C., Simmons, A., Feinstein, J. S., and Stein, M. B. (2003). Increased activation in the right insula during risk-taking decision making is related to harm avoidance and neuroticism. Neuroimage 19, 1439-1448. doi: 10.1016/S1053-8119(03)00251-9

Powell, M., and Ansic, D. (1997). Gender differences in risk behaviour in financial decision-making: an experimental analysis. J. Econ. Psychol. 18, 605-628. doi: 10.1016/S0167-4870(97)00026-3

Qi, H. X., Lyon, D. C., and Kaas, J. H. (2002). Cortical and thalamic connections of the parietal ventral somatosensory area in marmoset monkeys (Callithrix jacchus). J. Comp. Neurol. 443, 168-182. doi: 10.1002/cne.10113

Raichle, M. E. (2010). Two views of brain function. Trends Cogn. Sci. 14, 180-190. doi: 10.1016/j.tics.2010.01.008

Raichle, M. E., and Mintun, M. A. (2006). Brain work and brain imaging. Annu. Rev. Neurosci. 29, 449-476. doi: 10.1146/annurev.neuro.29.051605.112819
Seeley, W. W., Menon, V., Schatzberg, A. F., Keller, J., Glover, G. H., Kenna, H., et al. (2007). Dissociable intrinsic connectivity networks for salience processing and executive control. J. Neurosci. 27, 2349-2356. doi: 10.1523/JNEUROSCI.558706.2007

Skeel, R. L., Pilarski, C., Pytlak, K., and Neudecker, J. (2008). Personality and performance-based measures in the prediction of alcohol use. Psychol. Addict. Behav. 22, 402-409. doi: 10.1037/0893-164X.22.3.402

Smith, S. M., Fox, P. T., Miller, K. L., Glahn, D. C., Fox, P. M., Mackay, C. E., et al. (2009). Correspondence of the brain's functional architecture during activation and rest. Proc. Natl. Acad. Sci. U.S.A. 106, 13040-13045. doi: 10.1073/pnas.0905267106

Tobler, P. N., O'Doherty, J. P., Dolan, R. J., and Schultz, W. (2007). Reward value coding distinct from risk attitude-related uncertainty coding in human reward systems. J. Neurophysiol. 97, 1621-1632. doi: 10.1152/jn.00745.2006

Tomasi, D., and Volkow, N. D. (2010). Functional connectivity density mapping. Proc. Natl. Acad. Sci. U.S.A. 107, 9885-9890. doi: 10.1073/pnas.1001414107

Tomasi, D., and Volkow, N. D. (2012a). Abnormal functional connectivity in children with attention-deficit/hyperactivity disorder. Biol. Psychiatry 71, 443-450. doi: 10.1016/j.biopsych.2011.11.003

Tomasi, D., and Volkow, N. D. (2012b). Aging and functional brain networks. Mol. Psychiatry 17, 549-458. doi: 10.1038/mp.2011.81

Tomasi, D., and Volkow, N. D. (2012c). Gender differences in brain functional connectivity density. Hum. Brain Mapp. 33, 849-860. doi: 10.1002/hbm 21252

Tomasi, D., and Volkow, N. D. (2012d). Laterality patterns of brain functional connectivity: gender effects. Cereb. Cortex 22, 1455-1462. doi: 10.1093/cercor/bhr230

Vogt, B. A., Pandya, D. N., and Rosene, D. L. (1987). Cingulate cortex of the rhesus monkey: I. Cytoarchitecture and thalamic afferents. J. Comp. Neurol. 262, 256-270. doi: 10.1002/cne.902620207

Wang, X. T., Kruger, D. J., and Wilke, A. (2009). Life history variables and risk-taking propensity. Evol. Hum. Behav. 30, 77-84. doi: 10.1016/j.evolhumbehav.2008.09.006

Weber, E. U., Blais, A. R., and Betz, N. E. (2002). A domain-specific risk-attitude scale: measuring risk perceptions and risk behaviors. J. Behav. Decis. Making 15, 263-290. doi: 10.1002/bdm.414

Zou, Q., Ross, T. J., Gu, H., Geng, X., Zuo, X. N., Hong, L. E., et al. (2013). Intrinsic resting-state activity predicts working memory brain activation and behavioral performance. Hum. Brain Mapp. 34, 3204-3215. doi: 10.1002/hbm.22136

Zuckerman, M., Eysenck, S., and Eysenck, H. J. (1978). Sensation seeking in England and America: cross-cultural, age, and sex comparisons. J. Consult. Clin. Psychol. 46, 139-149. doi: 10.1037/0022-006X.46.1.139

Zuo, X. N., Ehmke, R., Mennes, M., Imperati, D., Castellanos, F. X., Sporns, O., et al. (2012). Network centrality in the human functional connectome. Cereb. Cortex 22, 1862-1875. doi: 10.1093/cercor/bhr269

Zuo, X. N., Kelly, C., Di Martino, A., Mennes, M., Margulies, D. S., Bangaru, S., et al. (2010). Growing together and growing apart: regional and sex differences in the lifespan developmental trajectories of functional homotopy. J. Neurosci. 30, 15034-15043. doi: 10.1523/JNEUROSCI.2612-10.2010

Conflict of Interest Statement: The authors declare that the research was conducted in the absence of any commercial or financial relationships that could be construed as a potential conflict of interest.

Received: 21 October 2013; accepted: 03 January 2014; published online: 28 January 2014.

Citation: Zhou Y, Li S, Dunn J, Li H, Qin W, Zhu M, Rao L-L, Song M, Yu C and Jiang $T$ (2014) The neural correlates of risk propensity in males and females using resting-state fMRI. Front. Behav. Neurosci. 8:2. doi: 10.3389/fnbeh.2014.00002 This article was submitted to the journal Frontiers in Behavioral Neuroscience. Copyright (C) 2014 Zhou, Li, Dunn, Li, Qin, Zhu, Rao, Song, Yu and Jiang. This is an open-access article distributed under the terms of the Creative Commons Attribution License (CC BY). The use, distribution or reproduction in other forums is permitted, provided the original author(s) or licensor are credited and that the original publication in this journal is cited, in accordance with accepted academic practice. No use, distribution or reproduction is permitted which does not comply with these terms. 


\section{APPENDIX}

Table A1 | Descriptive statistics of the assessment of risky propensity.

\begin{tabular}{lrr}
\hline & Mean & SD \\
\hline RPS & 3.2 & 1.2 \\
BIS_ATT & 16.2 & 3.1 \\
BIS_MOT & 21.7 & 3.6 \\
BIS_NP & 25.3 & 4.2 \\
SSS_TAS & 4.6 & 2.1 \\
SSS_ES & 3.1 & 1.7 \\
SSS_DIS & 4.1 & 2.0 \\
SSS_BS & 3.0 & 1.6 \\
TPQ_NS & 14.0 & 5.1 \\
TPQ_HA & 14.9 & 6.4 \\
TPQ_RD & 19.0 & 3.5 \\
EPQ_P & 4.4 & 2.8 \\
EPQ_E & 12.3 & 5.0 \\
EPQ_N & 10.9 & 5.4 \\
EPQ_L & 9.8 & 3.5 \\
\hline
\end{tabular}

Abbreviations: BIS_ATT, attentional factors of the Barratt Impulsiveness Scale; BIS_MOT, motor factors of the Barratt Impulsiveness Scale; BIS_NP, non-planning impulsiveness factors of the Barratt Impulsiveness Scale; EPQ_E, extraversion-introversion subscale of the Eysenck Personality Questionnaire; EPQ_N, neuroticism subscale of the Eysenck Personality Questionnaire; EPQ_P, psychotism subscale of the Eysenck Personality Questionnaire; RPS, Risk Propensity Scale; SSS_TAS, thrill and adventure seeking dimention of the Sensation Seeking Scale; SSS_ES, experience seeking dimention of the Sensation Seeking Scale; SSS_DIS, disinhibition dimention of the Sensation Seeking Scale; SSS_BS, boredom susceptibility dimention of the Sensation Seeking Scale; TPQ_NS, novelty seeking subscale of the Tridimensional Personality Questionnaire; TPQ_HA, harm avoidance subscale of the Tridimensional Personality Questionnaire; TPQ_RD, reward dependence subscale of the Tridimensional Personality Questionnaire.

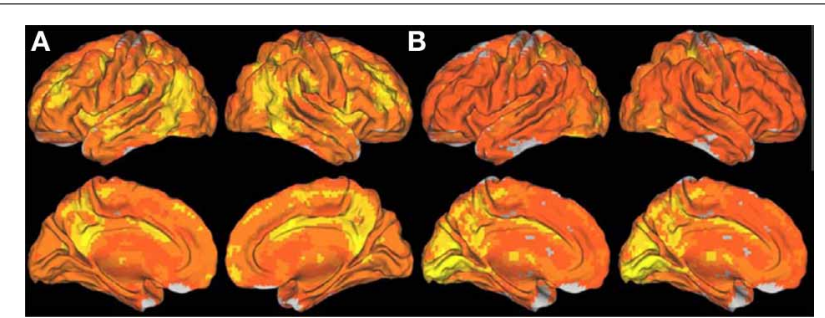

FIGURE A1 | Surface rendering showing the spatial distribution of the long- (A) and short-range (B) FCD in the human brain, which reflect the average number of functional connections per voxel across 289 participants. The images were created using the Computerized Anatomical Reconstruction and Editing Toolkit (CARET) 5.62, which is a free software developed at Washington University (http://brainvis.wustl.edu/wiki/index.php/Caret:About).

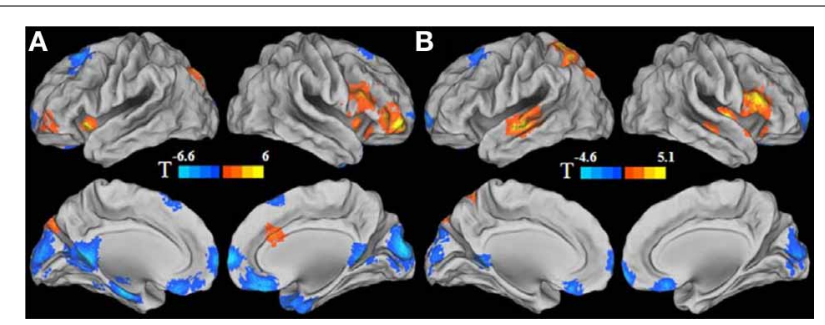

FIGURE A2 | Gender effect for long-range FCD (A) and for short-range FCD (B). For the purpose of presentation, cool color represents the regions showing reduced long- or short-range FCD in men, and warm color represents the regions showing reduced long- or short-range FCD in women. Statistical values were $T$ values for the simple effects. 

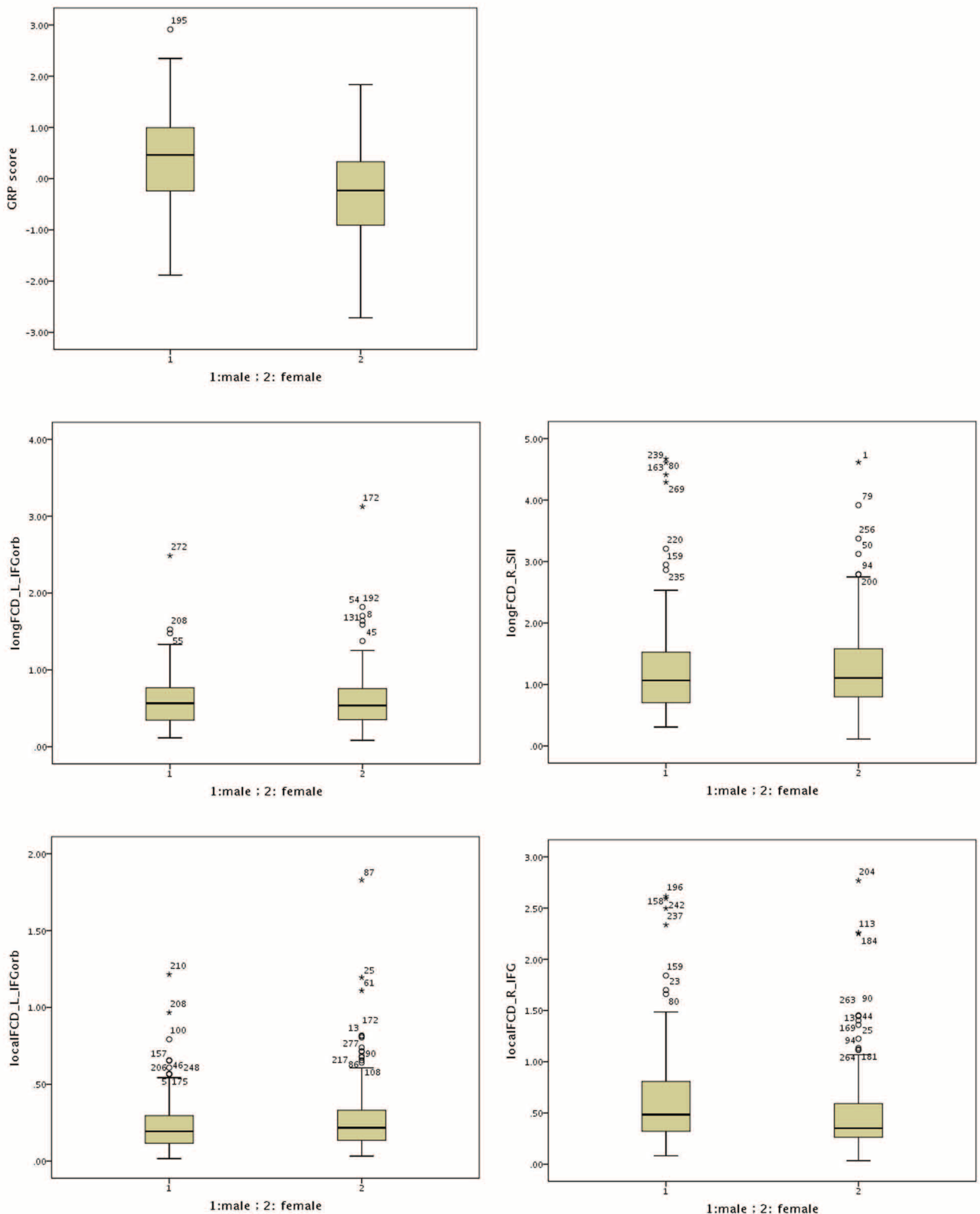

FIGURE A3 | Boxplots for the GRP score and the functional connectivity density (FCD) in males and females. Open dots indicate a mild outlier with scores more than $1.5 *$ IQR from the rest of the

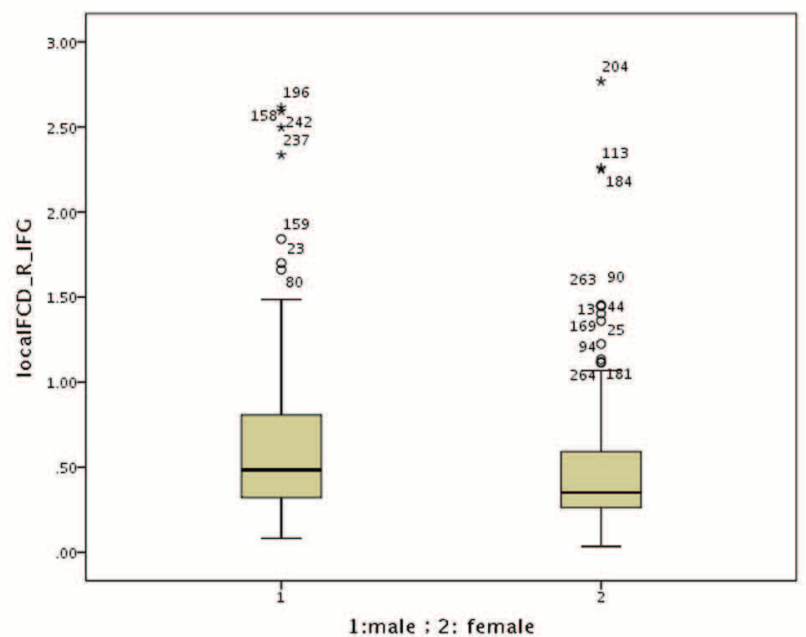

scores. Stars indicate an extreme outlier with scores more than $3 *$ IQR from the rest of the scores. IQR stands for interquartile range and is the middle $50 \%$ of the scores. 